\title{
Nonlinear diffraction of water waves: a classical perturbation method
}

\author{
M. Rahman ${ }^{1}$, S. H. Mousavizadegan ${ }^{2}$ \& D. Bhatta ${ }^{3}$ \\ ${ }^{1}$ Faculty of Computer Science, Dalhousie University, Halifax, Canada \\ ${ }^{2}$ Faculty of Marine Technology, Amirkabir University of Technology, Iran \\ ${ }^{3}$ The University of Texas Pan-American, Edinburg, USA
}

\begin{abstract}
This paper deals with the classical theory of the perturbation method to determine the solution of wave forces on a circular cylinder in regular waves. Nonlinear diffraction of water waves is considered here to demonstrate the powerful perturbation technique. The solution obtained by this method is compared with the available experimental data. It is found that the analytical solution agrees with the experimental data quite well. When we talk about wave effects on offshore structures, it is necessary to distinguish between small and large dimensions in relation to the characteristic wavelength and the wave amplitude. It is well known that the Morison equation displayed an empirical relationship in terms of the coefficient of mass, $C_{M}$, and of the coefficient of drag, $C_{D}$, the two hydrodynamic coefficients used to calculate wave forces on a small submerged cylinder. This relationship involves the inertia force and viscous drag force on the cylinder and assumes that the object is small so as not to disturb the incident wave field. However, as the diameter of the cylinder becomes large compared to the incident wavelength, the Morison equation does not apply and a diffraction theory must be used. In this case, viscous drag forces are assumed to be insignificant for smooth dimension structures (cylinders) and the inertia forces predominate. In this paper we discuss the theoretical formulation of second order wave loads. We have obtained the mathematical expressions for the forces and moments to predict the wave loads on large monolithic offshore structures.

Keywords: Morison equation, inertia force, drag force, nonlinear diffraction, radiation, wave forces, moments, perturbation method, second-order theory, offshore structures, cylinders, diffracted waves, incident waves.
\end{abstract}




\section{Introduction}

The determination of wave forces on offshore structures is essential to study the effects of waves, wind and current on them. The offshore structures should experience minimal movement to provide a stable work station for operations such as drilling and production of oil. The solution to the problem of ocean wave interaction with offshore structures is usually very complex. In many cases, only an approximate solution is sought. Some of the mathematical techniques required for the hydrodynamic problem associated with the design of offshore structures are analytical while many are numerical in nature. While the evolution of computers has made the numerical methods more advantageous over the classical analytical methods, numerical methods alone cannot find absolute success without being complemented by either analytical methods or at least experiments; in this sense analytical methods become a cost efficient and handy technique for designers in most cases.

Morison et al. [1] gave an empirical relationship in terms of coefficient of mass, $C_{M}$, and of coefficient of drag, $C_{D}$, the two hydrodynamic coefficients to calculate wave forces on a small submerged cylinder in regular waves. However, when the size of the structure is large compared to the wavelength, there are considerable scattering and diffraction in the wave pattern and in that situation the Morison equation is not suitable to calculate the wave loads and we must use the diffraction theory. A linear diffraction theory was first formulated by MacCamy and Fuchs [2] to calculate the wave loads on a vertical circular cylinder. The cylinder extends from well above the surface to the bottom of the sea. A number of investigators including Mogridge and Jamieson [3] have used this theory to obtain the wave loads on large submerged cylinders in the sea. In all these investigations, the analyses were restricted to linear theory only, which has very limited applications in a practical field because the character of the ocean waves is very often nonlinear. To improve the correlation between experimental data and theory, it is necessary to include the nonlinear effects in the diffraction theory.

Taking into consideration this motivation, many investigators have directed their attention towards nonlinear theory in water waves. Chakrabarti [4] obtained an expression for wave forces on a cylinder using Stokes's fifth order theory, but the kinematic boundary condition was not satisfied in the vicinity of the cylinder. The results obtained were compared with the second order theory of Yamaguchi and Tsuchiya [5] and he concluded that his theory and experimental showed good agreement with their experimental data. Raman and Venkatanarasaiah [6] obtained a nonlinear solution using a perturbation technique. Unfortunately, their experimental results were obtained using a relatively small diameter cylinder, for which the previous workers had assumed the viscous drag forces to be significant.

This paper deals with the theory of second-order wave load expressions to predict the wave forces and moments on large monolithic offshore structures. The theory is applied to a surface-piercing cylinder in regular waves. Following Stoker [7], we have developed a mathematical model of the nonlinear wave theory to correlate with nature. A perturbation technique has been used to solve the 
nonlinear diffraction problem. For the case of fixed offshore structures, closed form solutions for drift forces and moments have been obtained using the secondorder theory, and the predictions have been compared with available experimental data.

\section{Mathematical formulation}

A rigid vertical surface-piercing circular cylinder of radius $a$ and the diameter $D=2 a$ is acted upon by a train of regular surface waves of height $H$, progressing in the positive $x$ - direction (Figure 1). It is assumed that the fluid is incompressible and the motion irrotational. When the amplitude is large, the small amplitude theory does not hold good. In practice, the finite amplitude wave theory, namely the nonlinear wave theory, is of primary importance. In linear wave, the wave amplitudes to the second and higher orders are considered negligible, whereas in the finite amplitude wave theory these higher order terms are retained so as to give an accurate representation of the wave motion.

Let $\phi(r, \theta, z, t)$ denote the total fluid velocity potential and let $z=\eta(r, \theta, t)$ be the equation of the free surface, where $(r, \theta, z)$ are the cylindrical coordinates. Then everywhere in the region of the flow, the fluid motion is governed by Laplace's equation.

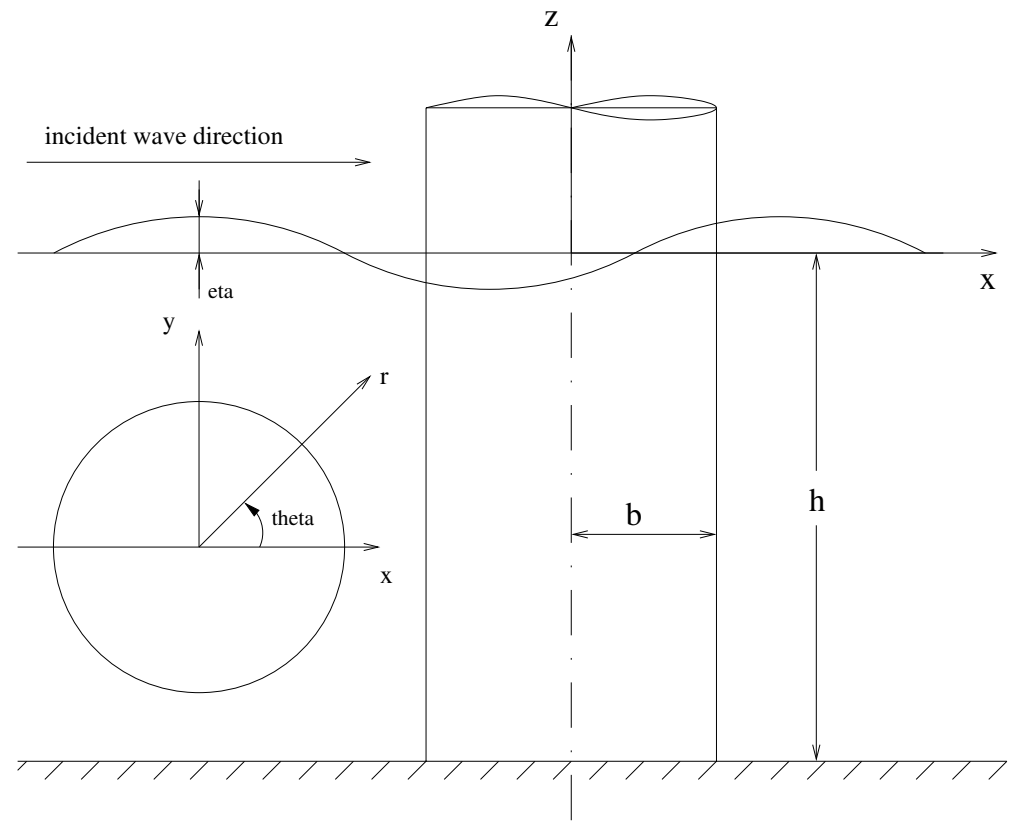

Figure 1: Definition sketch for a surface-piercing circular cylinder in regular waves. 
The motion is governed by the equation:

$$
\nabla^{2} \phi=\frac{\partial^{2} \phi}{\partial r^{2}}+\frac{1}{r} \frac{\partial \phi}{\partial r}+\frac{1}{r^{2}} \frac{\partial^{2} \phi}{\partial \theta^{2}}+\frac{\partial^{2} \phi}{\partial z^{2}}=0
$$

in the region

$$
a \leq r<\infty ;-h \leq z<\eta ;-\pi \leq \theta \leq \pi .
$$

Here $\nabla$ is the Laplacian operator. Equation (1) is Laplace's equation and it is derived from the fluid continuity equation. The boundary conditions associated with the equation (1) are as follows:

\section{Bottom boundary condition:}

Assuming the floor of the ocean to be flat, the boundary condition at the ocean bottom states that the vertical component of the velocity is zero at the bottom

$$
\frac{\partial \phi}{\partial z}=0 \text { on } z=-h
$$

\section{Body surface boundary condition:}

On the surface of the body the velocity of the fluid must be equal to zero

$$
\frac{\partial \phi}{\partial r}=0 \text { on } r=a ;-h \leq z \leq \eta
$$

\section{Dynamic free surface boundary condition:}

The dynamic free surface condition is derived from the Bernoulli equation, on the assumption that the atmospheric pressure outside the fluid is constant.

$$
\frac{\partial \phi}{\partial t}+g \eta+\frac{1}{2}\left[\left(\frac{\partial \phi}{\partial r}\right)^{2}+\left(\frac{1}{r} \frac{\partial \phi}{\partial \theta}\right)^{2}+\left(\frac{\partial \phi}{\partial z}\right)^{2}\right]=0 ; \quad z=\eta, \quad r \geq a .
$$

\section{Kinematic free surface boundary condition:}

The kinematic condition states that a particle lying on the free surface will continue to remain on the surface. Mathematically,

$$
\frac{\partial \eta}{\partial t}+\left(\frac{\partial \phi}{\partial r}\right)\left(\frac{\partial \eta}{\partial r}\right)+\frac{1}{r^{2}}\left(\frac{\partial \phi}{\partial \theta}\right)\left(\frac{\partial \eta}{\partial \theta}\right)=\frac{\partial \phi}{\partial z} ; \quad \text { on } z=\eta, \quad r \geq a
$$

In addition to the above boundary conditions, there is the Orr-Sommerfeld radiation condition to be satisfied by the scattering potential $\Phi_{s}$, where $\phi=$ $\operatorname{Re}\left\{\left(\Phi_{I}+\Phi_{s}\right) e^{-i \sigma t}\right\}$ in which $\Phi_{I}$ and $\Phi_{s}$ are defined to be the complex incident and complex scattered potentials respectively. This boundary condition states that the diffracted wave must vanish at infinity and mathematically can be defined by the Orr-Sommerfeld condition

$$
\lim _{r \rightarrow \infty} \sqrt{r}\left\{\frac{\partial}{\partial r} \pm i k\right\} \Phi_{s}=0,
$$

where $\sqrt{-1}=i$ and $k$ is the incident wave number. Here Re stands for the real part, $\sigma$ is the wave frequency. 
The velocity components in cylindrical coordinates are $\mathbf{V}=\nabla \phi$, i.e.

$$
v_{r}=\frac{\partial \phi}{\partial r}, v_{\theta}=\frac{1}{r} \frac{\partial \phi}{\partial \theta}, \text { and } v_{z}=\frac{\partial \phi}{\partial z},
$$

where $\phi$ is the total velocity potential, $\eta$ is the height of the free surface, $h$ is the depth of water below the still water level, $v_{r}, v_{\theta}$ and $v_{z}$ are the velocity components, $g$ is the acceleration due to gravity, $k$ is the incident wave number $(=$ $2 \pi / L), x, y, z$ are rectangular coordinates, $r, \theta, z$ are the cylindrical coordinates, $t$ is the time variable and $L$ is the wavelength. The Cartesian form of (1) is

$$
\nabla^{2} \phi=\frac{\partial^{2} \phi}{\partial x^{2}}+\frac{\partial^{2} \phi}{\partial y^{2}}+\frac{\partial^{2} \phi}{\partial z^{2}}=0
$$

where $x=r \cos \theta, y=r \sin \theta$ and $z=z$.

The formulas for the forces $\mathbf{F}$ and moments $\mathbf{M}$ of the fluid on the body $S$ can be written in vector form as follows:

$$
\left\{\begin{array}{c}
\mathbf{F} \\
\mathbf{M}
\end{array}\right\}=\iint_{S} P\left\{\begin{array}{c}
\mathbf{n} \\
\mathbf{r} \times \mathbf{n}
\end{array}\right\} d S
$$

where $\mathbf{n}$ is the outward normal vector from the body surface, $P$ is the pressure field which can be obtained from the Bernoulli's equation as illustrated in the next section and $\mathbf{r}$ is the radial distance vector.

\section{Perturbation method of solution}

The problem is to work with the complete form of the equation to assume that the solution can be represented in terms of a power series expansion of the parameter $\varepsilon$ where $\varepsilon=k H / 2=k A$. $A$ is the amplitude of the wave. Thus expanding $\phi, \eta$ and $P$ as a series in powers of $\varepsilon$ yields

$$
\begin{aligned}
& \phi=\sum_{n=1}^{\infty} \varepsilon^{n} \phi_{n}, \\
& \eta=\sum_{n=1}^{\infty} \varepsilon^{n} \eta_{n}, \\
& P=\sum_{n=0}^{\infty} \varepsilon^{n} P_{n} .
\end{aligned}
$$

The sum of the terms up to index $n$ represents the $n t h$-order theory for any particular quantity, $P$ represents the pressure field, and $\phi, \eta$ and $P$ are all functions 
of $x, y, z$ and $t$. At the free surface, $z=\eta(x, y, t)$, and so we have

$$
\phi(x, y, z, t)=\phi[x, y, \eta(x, y, t), t] .
$$

The modified velocity potential at the free surface is

$$
\phi(x, y, \eta, t)=\varepsilon \phi_{1}(x, y, 0, t)+\varepsilon^{2}\left[\phi_{2}(x, y, 0, t)+\eta_{1}\left(\frac{\partial \phi_{1}}{\partial z}\right)_{z=0}\right]+O\left(\varepsilon^{3}\right) .
$$

Substituting the series into the dynamic and kinematic free surface boundary conditions, it is found that the first-order potential $\phi_{1}$ and the second-order potential $\phi_{2}$ satisfy the following equations, respectively:

$$
\begin{aligned}
\frac{\partial^{2} \phi_{1}}{\partial t^{2}}+g \frac{\partial \phi_{1}}{\partial z}=0 \quad \text { on } z=0 & \\
\frac{\partial^{2} \phi_{2}}{\partial t^{2}}+g \frac{\partial \phi_{2}}{\partial z}= & -\eta_{1} \frac{\partial}{\partial z}\left\{\frac{\partial^{2} \phi_{1}}{\partial t^{2}}+g \frac{\partial \phi_{1}}{\partial z}\right\} \\
& -\frac{\partial}{\partial t}\left\{\left(\frac{\partial \phi_{1}}{\partial x}\right)^{2}+\left(\frac{\partial \phi_{1}}{\partial y}\right)^{2}+\left(\frac{\partial \phi_{1}}{\partial z}\right)^{2}\right\}, \quad r \geq a .
\end{aligned}
$$

The pressure $P(r, \theta, z, t)$ may be determined from the Bernoulli's equation and on substitution for $\phi$ as a series in power of $\varepsilon$, leads to

$$
\begin{aligned}
P= & -\rho g z-\varepsilon \rho \frac{\partial \phi_{1}}{\partial t}-\varepsilon^{2} \rho\left[\frac{\partial \phi_{2}}{\partial t}+\frac{1}{2}\left(\left(\frac{\partial \phi_{1}}{\partial r}\right)^{2}\right.\right. \\
& \left.\left.\left.\left.+\frac{\partial \phi_{1}}{\partial z}\right)^{2}+\frac{1}{r^{2}} \frac{\partial \phi_{1}}{\partial \theta}\right)^{2}\right)\right]+O\left(\varepsilon^{3}\right) .
\end{aligned}
$$

The total horizontal force is

$$
F_{x}=\int_{0}^{2 \pi} \int_{-h}^{\eta}|P|_{r=a}(-a \cos \theta) d z d \theta .
$$

Now let us write $F_{x}$ as a perturbation series up to order $\varepsilon^{2}$ as follows:

$$
F_{x}=\varepsilon F_{x 1}+\varepsilon^{2} F_{x 2},
$$

where the first order contribution is

$$
\varepsilon F_{x 1}=a \rho \int_{0}^{2 \pi}\left\{\int_{-h}^{0}\left(\varepsilon \frac{\partial \phi_{1}}{\partial t}\right)_{r=a}\right\} \cos \theta d \theta
$$

and the second-order contribution is

$$
\begin{aligned}
\varepsilon^{2} F_{x 2}= & a \rho \int_{0}^{2 \pi}\left[\int_{0}^{\varepsilon \eta}\left(g z+\varepsilon \frac{\partial \phi_{1}}{\partial t}\right)_{r=a} d z\right. \\
& \left.+\varepsilon^{2} \int_{-h}^{0}\left\{\frac{\partial \phi_{2}}{\partial t}+\frac{1}{2}\left(\frac{\partial \phi_{1}}{\partial z}\right)^{2}+\frac{1}{2 a^{2}}\left(\frac{\partial \phi_{1}}{\partial \theta}\right)^{2}\right\}_{r=a} d z\right] \cos \theta d \theta
\end{aligned}
$$


It is to be noted here that in the second-order force evaluation given in (19), the first term on the right can be defined as the waterline force, the third term as the dynamic force and the second term as the quadratic force as stated by Lighthill [8].

The expressions for $\phi_{1}$ and $\phi_{2}$ are given as follows (Rahman [9]):

$$
\begin{aligned}
& \phi_{1}=\frac{\sigma \cosh k(z+h)}{k^{2} \sinh k h} e^{-i \sigma t} \sum_{m=0}^{\infty} \delta_{m} i^{m} A_{m}(k r) \cos m \theta, \\
& \phi_{2}=\frac{\sigma e^{-2 i \sigma t}}{2 k^{2}} \sum_{m=0}^{\infty}\left\{\int_{0}^{\infty} D_{m}\left(k_{2}\right) A_{m}\left(k_{2} r\right) \cosh k_{2}(z+h) d k_{2}\right\} \cos m \theta .
\end{aligned}
$$

with the dispersion relation which is $\sigma^{2}=g k \tanh k h$.

\section{Forces and moments on the circular cylinder}

Using the results presented by Rahman and Heaps [10], the non-dimensional forms of the first-order and second-order components of the total forces may now be written as

$$
\frac{F_{x 1}}{\rho g D^{3}}=\frac{\tanh k h}{2(k a)^{3}} \cdot \frac{\cos \left(\sigma t-\alpha_{1}\right)}{\left|H_{1}^{(1)^{\prime}}(k a)\right|},
$$

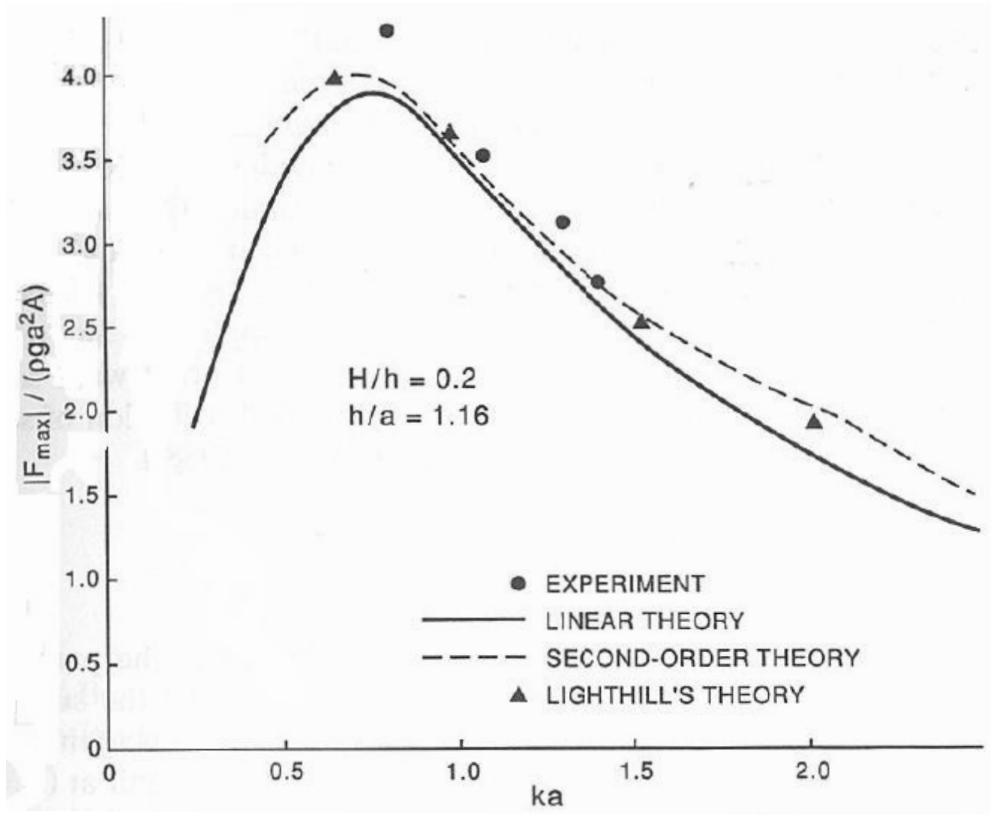

Figure 2: Comparison of linear and second-order wave forces with the experimental data of Chakrabarti [4]. 


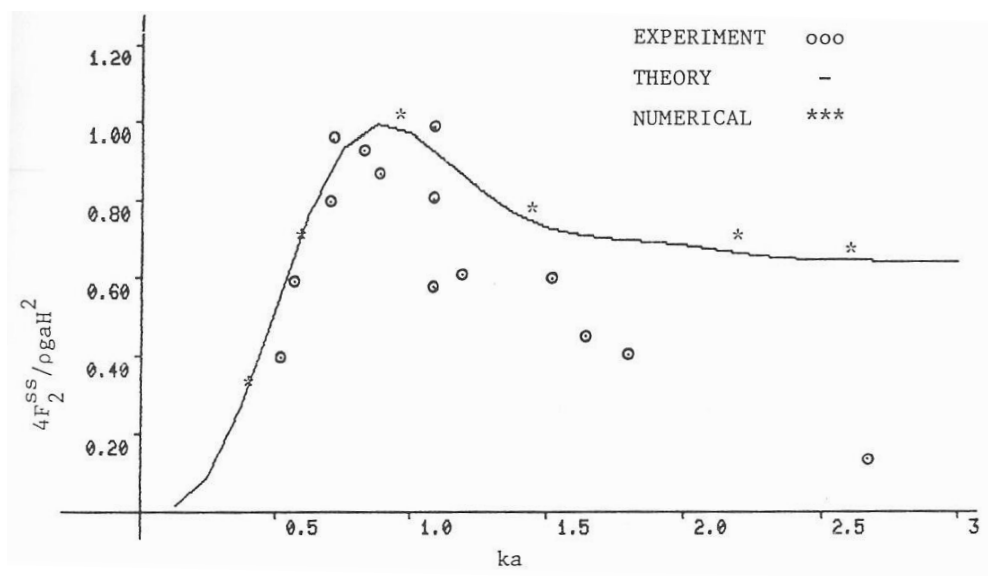

Figure 3: Potential drift force on fixed circular cylinders, $h / a=1.16$ : Comparison with the numerical data of Garrison [11].

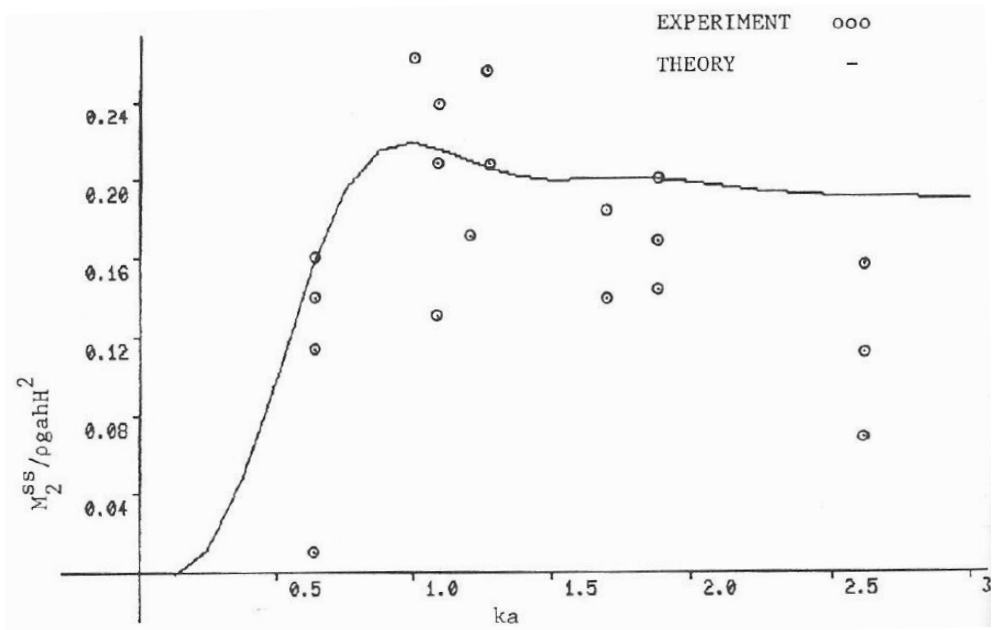

Figure 4: Potential drift moment on fixed circular cylinders, $h / a=1.16$ : Comparison with the numerical data of Garrison [11].

and

$$
\begin{aligned}
\frac{F_{x 2}}{\rho g D^{3}}= & \left\{\frac{e^{-2 i \sigma t} \tanh k h}{8(k a)} \int_{0}^{\infty} G\left(k_{2}\right) d k_{2}+c . c .\right\} \\
& -\frac{1}{4 \pi(k a)^{4}} \sum_{\ell=0}^{\infty}(-1)^{\ell}\left[\left(3-\frac{2 k h}{\sinh 2 k h}\right)+\frac{\ell(\ell+1)}{a^{2} k^{2}}\right. \\
& \left.\times\left(1+\frac{2 k h}{\sinh 2 k h}\right)\right] \times\left[C_{\ell} \cos 2 \sigma t-S_{\ell} \sin 2 \sigma t\right] \\
& -\frac{1}{4 \pi(k a)^{4}} \sum_{\ell=0}^{\infty}\left[\left(1-\frac{\ell(\ell+1)}{a^{2} k^{2}}\right)\left(1+\frac{2 k h}{\sinh 2 k h}\right) E_{\ell}\right]
\end{aligned}
$$




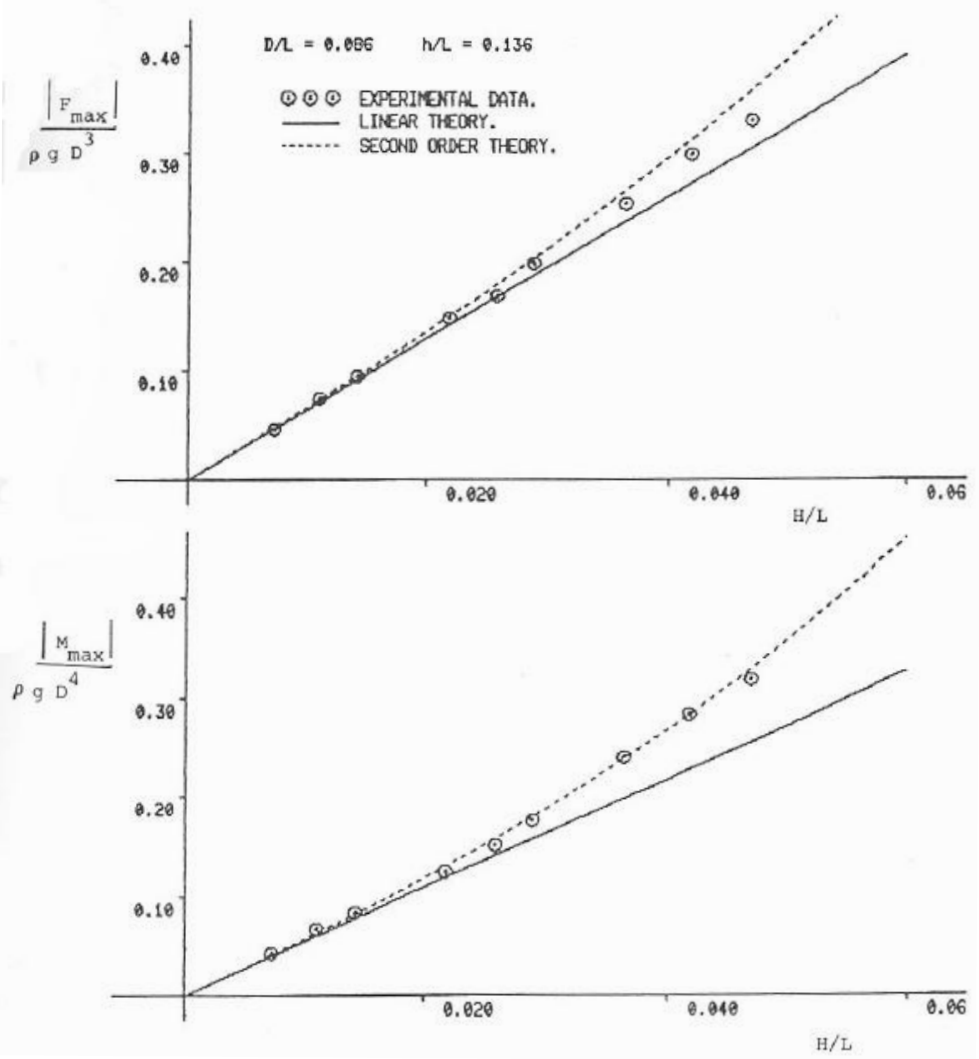

Figure 5: Comparison of predicted and measured forces and moments on circular cylinders (Experimental data of Mogridge and Jamieson [3]).

where

$$
G\left(k_{2} a\right)=\frac{\int_{k a}^{\infty} A_{1}\left(k_{2} r\right) B_{1}(k r) d(k r)}{\left(k_{2} a\right)\left[\left(k_{2} a\right)-\frac{4 k a \tanh k h}{\tanh k_{2} h}\right] H_{1}^{(1)^{\prime}}\left(k_{2} a\right)} .
$$

The second-order force (21) contains two parts; one oscillatory and the other steady state. They are defined, respectively, as

$$
\frac{F_{x 2}}{\rho g D^{3}}=\frac{F_{2}^{O S}}{\rho g D^{3}}+\frac{F_{2}^{S S}}{\rho g D^{3}} .
$$

Similarly, the non-dimensional forms of the linear and of the second-order moments are given by

$$
\frac{\varepsilon M_{1}}{\rho g D^{4}}=C_{M} \frac{(H / L) \pi}{16(D / L)(k a)}[k h \tanh k h+\operatorname{sech} k h-1] \cos \left(\sigma t-\alpha_{1}\right)
$$


and

$$
\begin{aligned}
\frac{\varepsilon^{2} M_{2}}{\rho g D^{4}}= & \frac{(H / L)^{2}(h / D)}{4 \pi^{3}(D / L)^{4}} \sum_{\ell=0}^{\infty}\left[\frac{\ell(\ell+1)}{k^{2} a^{2}} B-C\right] E_{\ell} \\
& +\left[\frac{(H / L)^{2}(h / D)}{8(D / L)^{2}} \tanh k h e^{-2 i \sigma t} \int_{0}^{\infty} G\left(k_{2} a\right) \lambda\left(k_{2} h\right) d\left(k_{2} a\right)+\text { c.c. }\right] \\
& -\frac{(H / L)^{2}(h / D)}{4 \pi^{3}(D / L)^{4}} \sum_{\ell=0}^{\infty}(-1)^{\ell+1}\left[A+\frac{\ell(\ell+1)}{k^{2} a^{2}} B\right] \\
& \times\left[C_{\ell} \cos 2 \sigma t-S_{\ell} \sin 2 \sigma t\right] .
\end{aligned}
$$

The second-order moment (24) contains two parts; one oscillatory and the other steady state. They are defined, respectively, as

$$
\frac{M_{2}}{\rho g D^{4}}=\frac{M_{2}^{O S}}{\rho g D^{4}}+\frac{M_{2}^{S S}}{\rho g D^{4}} .
$$

The graphical representations of these results are displayed with the comparison of the experimental and numerical data. It is worth mentioning here that all the parameters defined here are found in the work of Rahman [9].

\section{Results and conclusions}

The analytical and numerical and experimental results are displayed and discussed in this section. In Fig. 2, both the first-order and the second-order solutions (see Rahman [9]) are compared with the force measurements of Chakrabarti [4], which are generally seen to be closer to the second-order predictions. The normalized dynamic drift forces and moments are potted against the diffraction parameter $k a$ in Fig. 3 and Fig. 4 using the analytical results. This results are compared with the numerical solutions obtained by Garrison [11] and the present predicted results quite agree with the Garrison's numerical data. Garrison used the Green function method to determine the drift forces and moments. In Fig. 5, analytical solutions are compared with the experimental data of Mogridge and Jamieson [3] for a vertical circular cylindrical structure. These results were computed taking the averages of the positive and negative maximum loads. The theoretical predictions of moments seem to agree very well with the measured values for the given range of parameters.

\section{Acknowledgements}

We are grateful to the Natural Sciences and Engineering Research Council of Canada for its financial support leading to this paper. We are also thankful to the Faculty of Computer Science at Dalhousie University for the computer facilities provided to complete this paper. 


\section{References}

[1] Morison, J. R., O’Brien, M. P., Johnson, J. W. and Schaaf, S. A. (1950). The forces exerted by surface waves on piles, J. Petrol. Technology, 186, 149154.

[2] MacCamy, R. C. and Fuchs, R. A. (1954). Wave forces on piles: a diffraction theory, U. S. Army Beach Erosion Board, Techn. Memo. No. 69, December, 17 pages.

[3] Mogridge, G. R. and Jamieson, W. W. (1975). Wave forces on circular caissons: theory and experiment, Can. J. Civ. Engg., 2, 540-548.

[4] Chakrabarti, S. K. (1975). Second-order wave forces on large vertical cylinders, Proc. ASCE, 101, WW3, 311-317.

[5] Yamaguchi, M and Tsuchiya, Y. (1974). Nonlinear effects of wave pressures and wave forces on a large cylindrical pile, Proc. Civ. Engg. Soc. in Japan, 229, 41-53 (in Japanese).

[6] Raman, H. and Venkatanarasaiah, P. (1976). Forces due to nonlinear water waves on vertical cylinders, Proc. ASCE, 102, WW3, 301-316.

[7] Stoker, J. J. (1957). Water waves, Interscience, New York.

[8] Lighthill, M. J. (1979). Waves and hydrodynamic loading, Proc. Second Int. Conf. on The Behaviour of Offshore Structures, BOSS'79, London, 1, 1-40.

[9] Rahman, M. (1988). The hydrodynamics of waves and tides with applications, Topics in Engineering Vol. 4. Edited by Carlos Brebbia and J. J. Connor, Computational Mechanics Publications, Southampton UK and Boston USA.

[10] Rahman, M. and Heaps, H. S. (1983). Wave forces on offshore structures: nonlinear wave diffraction by large cylinders, J. Physical Oceano., 13, 22252235.

[11] Garrison, C. J. (1984). Wave structure interaction, Proc. Specialty Conf. Comput. Methods in Offshore Engg., CSCE, Halifax, N.S., Canada, 1-72. 